\title{
Cetoacidosis Diabética y estado hiperglucémico hiperosmolar en COVID-19. Reporte de casos
}

\author{
Diabetic Ketoacidosis and hyperosmolar hyperglucemic state \\ in COVID-19. Case report \\ Sacoto Flores Goethe Salomón', Sánchez Coraizaca Galo Dario', Cajilema \\ Acero Luis Andrés', Sanango Jara María Ximena'
}

VOLUMEN 39 | N 1 | SEPTIEMBRE 2021

FECHA DE RECEPCIÓN: 04/04/2021 FECHA DE APROBACIÓN: 21/07/2021 FECHA PUBLICACIÓN: 16/09/2021

1. Hospital Homero Castanier
Crespo. Clínica.
Azogues - Ecuador

\section{Caso Clinical Clínico Case}

DOI: https://doi.org/10.18537/RFCM.39.01.05

Correspondencia:

goethe.sacotoducuenca.edu.ec

Dirección:

Miguel Díaz y Remigio Crespo

Código Postal:

010203

Celular:

0968730021

Cuenca - Ecuador

\section{RESUMEN}

Introducción: Diabetes Mellitus (DM) es un factor de riesgo independiente para predecir gravedad en infección por COVID-19.

Casos clínicos: se presentan cuatro casos de crisis hiperglucémicas tipo estado hiperosmolar no cetósico (EHNC) y cetoacidosis diabética (CAD) asociados a COVID-19, tres con antecedente de DM y uno con nuevo diagnóstico de DM. El desenlace fue fatal para los casos con CAD y el caso de EHNC fue dado de alta en condición estable.

Conclusión: es fundamental comprender las características de presentación de complicaciones agudas de la diabetes en pacientes con COVID-19 y sospechar este tipo de alteraciones metabólicas en infección por SARS-CoV-2, aspectos importantes para un diagnóstico y tratamiento oportuno, mejorando en gran medida el pronóstico de los pacientes.

Palabras clave: COVID-19, Diabetes Mellitus, cetoacidosis diabética.

\section{ABSTRACT}

Introduction: Introduction: Diabetes Mellitus (DM) is an independent risk factor to predict severity in COVID-19 infection.

Clinical cases: we present four cases of hyperglycemic seizures type hyperosmolar nonketotic state (EHNC) and diabetic ketoacidosis (DKA) associated with COVID-19, three with a history of DM and one with a new diagnosis of DM. The outcome was fatal for the CAD cases and the EHNC case was discharged in stable condition.

Conclusion: it is essential to understand the presentation characteristics of acute complications of diabetes in patients with COVID-19 and to suspect this type of metabolic alterations in SARS-CoV-2 infection, important aspects for a timely diagnosis and treatment, greatly improving the prognosis of patients.

Key words: COVID-19, Diabetes Mellitus, diabetic ketoacidosis. 


\section{INTRODUCCIÓN}

A inicios de diciembre de 2019 , se reportaron en Wuhan (China) 3 casos de neumonía atípica provocada por un coronavirus nuevo, denominado SARS-CoV-2, cuya enfermedad se conoce como COVID-191. La presencia de infecciones graves es prevalente en pacientes con diabetes mellitus $^{2}$ y esta se considera un factor de riesgo independiente para predecir gravedad en infección por COVID-193,4. En pacientes con DM pre-existente o de diagnóstico nuevo, el COVID-19 puede provocar crisis de hiperglucemia aguda tipo CAD y EHNC, empeorando el pronóstico ${ }^{5,6}$.

\section{PRESENTACIÓN DE CASOS}

Se realizó una descripción de cuatro casos con diagnóstico de COVID-19, luego de un test positivo para SARS-CoV-2 por reacción en cadena de la polimerasa con transcripción inversa en tiempo real (PCR-RT) o anticuerpos positivos y la presencia de crisis hiperglucémica tipo $C A D$ y EHNC. La presentación y evolución clínica, así como el manejo y exámenes complementarios fueron tomados de los registros médicos físicos y digitales de cada caso y revisados por los autores. La CAD fue definida como: $\mathrm{pH}$ arterial $<7.3 \mathrm{y} / \mathrm{o} \mathrm{HCO} 3$ en suero $<18 \mathrm{mmol} / \mathrm{L}$, cetonas en orina positivas, anión gap $>10$, estado mental alerta o alterado. EI EHNC como: $\mathrm{pH}$ arterial $>7.3, \mathrm{HCO} 3$ en suero $>18 \mathrm{mmol} / \mathrm{L}$, osmolaridad efectiva $>320 \mathrm{mOsm} / \mathrm{kg}$ y anión gap variable ${ }^{7}$. Las características demográficas, alteraciones analíticas y marcadores inflamatorios se detallan en las Tablas $\mathrm{N}^{\circ} 1$ y $\mathrm{N}^{\circ} 2$. En la Imagen $\mathrm{N}^{\circ} 1$ se describen los principales hallazgos radiológicos.

\section{Tabla $N^{\circ} 1$}

Características demográficas, alteraciones analíticas y marcadores inflamatorios

\begin{tabular}{|c|c|c|c|c|c|c|c|c|c|c|c|c|c|c|c|c|}
\hline 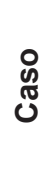 & $\begin{array}{l}\text { 윰 } \\
\text { 무 }\end{array}$ & 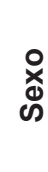 & 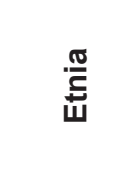 & $\mathscr{0}$ & 동 & $\begin{array}{l}\text { O̊ } \\
\text { ำ }\end{array}$ & ن & 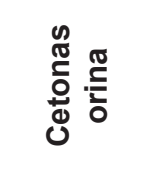 & $\stackrel{\text { D }}{E}$ & $\begin{array}{l}\frac{N}{0} \\
\square\end{array}$ & 志 & ด̣ & & ర్ల & I & 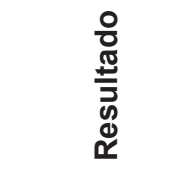 \\
\hline 1 & 86 & M & Mestiza & 687 & 7.45 & 25.6 & 28 & Negativo & 381 & 6.8 & 245 & 4.5 & 1261 & $\mathrm{Si}$ & 10 & Recuperado \\
\hline 2 & 61 & M & Mestiza & 433 & 7.25 & 10.6 & 26 & nd & 313 & 0.09 & nd & nd & nd & No & 1 & Fallece \\
\hline 3 & 67 & $\mathrm{~F}$ & Mestiza & 568 & 7.35 & 13.8 & 20 & +++ & 317 & 6.04 & 983 & 2.6 & 361.9 & $\mathrm{Si}$ & 6 & Fallece \\
\hline 4 & 33 & $\mathrm{~F}$ & Mestiza & 251 & 7.24 & 14.4 & 12.6 & ++ & 313 & 8 & 394 & 0.8 & 82 & $\mathrm{Si}$ & 13 & Fallece \\
\hline
\end{tabular}

GS: glucosa sanguínea, HCO3: bicarbonato, AG: anión gap, OsmS: Osmolaridad sérica, PCR: proteína C reactiva, LDH: lactato deshidrogenasa, D-D:dímero D, GC: glucocorticoide, EH: estancia hospitalaria/días. M: masculino, F: femenino, nd: no disponible.

*El glucocorticoide administrado fue dexametasona 6 mg día. 


\section{Tabla $\mathrm{N}^{\circ} 2$}

Estudios complementarios

\begin{tabular}{llllll}
\hline & \multicolumn{1}{c}{$\begin{array}{c}\text { Caso } 1 \\
\text { EHNC }\end{array}$} & $\begin{array}{c}\text { Caso 2 } \\
\text { CAD }\end{array}$ & $\begin{array}{c}\text { Caso } 3 \\
\text { CAD }\end{array}$ & \multicolumn{1}{c}{$\begin{array}{c}\text { Caso } 4 \\
\text { CAD }\end{array}$} & \multicolumn{1}{c}{ Referencia } \\
\hline Hemoglobina & 18.1 & 12.6 & 13.6 & 7.1 & g/dl 14-18 \\
Hematocrito & 56.2 & 37.2 & 39.2 & 23.5 & $40-54 \%$ \\
Leucocitos & 11.64 & 12.82 & 14.52 & 11.47 & $5.0-10.0 \times 10^{3} / \mathrm{mL}$ \\
Neutrófilos & 92 & 87.9 & 90.5 & 87.4 & $45-74 \%$ \\
Linfocitos & 5.8 & 4.4 & 6.7 & 7.8 & $16-45 \%$ \\
Plaquetas & 130 & 335 & 482 & 302 & $150-450 \times 10^{3} / \mathrm{mL}$ \\
Glucemia & 687 & 433 & 584 & 251.5 & $70-105 \mathrm{mg} / \mathrm{dL}$ \\
Urea & 222.1 & 28.5 & 102.5 & 33.3 & $19-44.1 \mathrm{mg} / \mathrm{dL}$ \\
Creatinina & 2.92 & 0.87 & 2.38 & 0.79 & $0.8-1.25 \mathrm{mg} / \mathrm{dL}$ \\
Na & 155 & 144.9 & 134 & 146.9 & $135-145 \mathrm{mEq} / \mathrm{L}$ \\
K & 4.7 & 3.8 & 3.5 & 4.6 & $3.5-5.1 \mathrm{mEq} / \mathrm{L}$ \\
AST & 17.4 & 486.3 & 24.9 & 38.1 & $05-34 \mathrm{U} / \mathrm{L}]$ \\
ALT & 38.5 & 320.3 & 584 & 22.5 & $05-55 \mathrm{U} / \mathrm{L}]$ \\
PCR & 6.8 & 0.09 & 102.5 & 8 & $<0.5 \mathrm{mg} / \mathrm{ml}$ \\
pH & 7.32 & 7.25 & 7.33 & 7.24 & $7.35-7.45$ \\
PO2 & 47 & 60 & 35 & 47.7 & $75-100 \mathrm{mmHg}$ \\
PCO2 & 36.9 & 32.9 & 25.2 & 32.4 & $35-45 \mathrm{mmHg}$ \\
HCO3 & 18.7 & 14.2 & 13.8 & 13.7 & $22-26 \mathrm{mmol} / \mathrm{L}$ \\
BE & -7.3 & -1.3 & -11.7 & -12.6 & $\mathrm{mmol} / \mathrm{L}$ \\
SATO2 & 80 & 87 & 90 & 74 & $\%$ \\
PO2/FiO2 & 128 & 134 & 91 & 136 & $>300 \mathrm{mmHg}$ \\
Lactato & 1.73 & 12.71 & 1.75 & 1.33 & $0.5-2.2 \mathrm{mmol} / \mathrm{L}$ \\
Anion gap & 26.6 & 17 & 20 & 20.9 & $7-14 \mathrm{mEq} / \mathrm{L}$ \\
Interleucina 6 & - & - & 194.6 & 13.8 & $<7 \mathrm{pg} / \mathrm{ml}$ \\
Procalcitonina & 0.27 & - & 0.45 & 0.27 & $<0.04 \mathrm{ng} / \mathrm{ml}$ \\
\hline & & & & &
\end{tabular}

Caso 1: hombre de 86 años, con antecedentes de hipotiroidismo, hipertensión arterial, falla cardiaca y EPOC oxígeno dependiente, acude por malestar general, anorexia, tos productiva, mialgias, alza térmica y disnea de pequeños esfuerzos. En la evaluación inicial se encontró deshidratado con pulso de 100 por minuto, tensión arterial de 80/40 $\mathrm{mmHg}, 34$ respiraciones minuto, temperatura de $36.1^{\circ} \mathrm{C}$ y saturación de oxígeno $92 \%$ ( $\mathrm{FiO} 2$ 0.28). Al examen físico, desorientado, mucosas orales secas. Pulmones con murmullo vesicular disminuido, estertores crepitantes bilaterales y difusos. Edema de extremidades con fóvea. En complementarios se reportaron: glucemia de 687 $\mathrm{mg} / \mathrm{dl}$, urea de $222 \mathrm{mg} / \mathrm{dl}$, creatinina $2.92 \mathrm{mg} /$ $\mathrm{dl}$, sodio sérico $155 \mathrm{mEq} / \mathrm{L}$, potasio sérico 4.7 $\mathrm{mEq} / \mathrm{L}$, osmolaridad efectiva mayor a $>320 \mathrm{mOsm} /$ $\mathrm{kg}$, gasometría arterial: $\mathrm{pH} 7.32$, bicarbonato $18.7 \mathrm{mmol} / \mathrm{L}$, resultados compatibles con EHNC; serología para SARS-CoV-2 positiva. Se inició hidratación parenteral, oxígeno por cánula nasal, insulinoterapia, corrección de hipernatremia, evidenciándose disminución progresiva de glucemia, natremia y normalización de creatinina, una vez estabilizado fue dado de alta con diagnóstico adicional de DM de novo.

Caso 2: hombre de 61 años, con antecedentes de DM tipo 2 (DM2) de 5 años de evolución sin tratamiento, referido por insuficiencia respiratoria, 
crisis convulsivas y pérdida súbita del estado de conciencia. En la evaluación inicial se encontró deshidratado, 105 latidos minuto, 36 respiraciones minuto, tensión arterial de 100/70 $\mathrm{mmHg}$, temperatura de $36^{\circ} \mathrm{C}$ y saturación de oxígeno 93\% ( $\mathrm{FiO} 2$ 0.44). Al examen físico, paciente en estado comatoso (Glasgow $7 / 15$ ), mucosa oral seca, patrón respiratorio superficial, con uso de musculatura accesoria, campos pulmonares hipoventilados, estertores crepitantes bilaterales con predominio basal. En complementarios se reportaron: leucocitosis, neutrofilia y linfopenia, glucemia de $433 \mathrm{mg} / \mathrm{dl}$, TGO: $486.3 \mathrm{U} / \mathrm{L}, \mathrm{TGP}: 320 \mathrm{U} / \mathrm{L}$, en gasometría arterial acidosis metabólica compensada con anión gap aumentado, osmolaridad efectiva de $313 \mathrm{mOsm} / \mathrm{kg}$, serología para SARS-CoV-2 positiva. Se inició protocolo terapéutico para cetoacidosis diabética, oxigenoterapia a flujo alto, antibioticoterapia empírica y profilaxis anticonvulsivante. Fue trasladado a unidad de cuidados intensivos para manejo invasivo de vía aérea; sin embargo, paciente falleció al persistir el deterioro clínico (metabólico y del estado ácidobase) y radiológico.

Caso 3: mujer de 67 años con antecedentes de hipertensión arterial y DM2 (tiempo de evolución desconocido), acudió por tos, disnea clase funcional III, astenia, dolor abdominal difuso, diarrea, edema generalizado, nistagmo y somnolencia. Al examen físico se reportó mucosa oral seca, nistagmo horizontal, tensión arterial de 135/111 mmHg, 69 latidos minuto, 24 respiraciones minuto, temperatura $36.5{ }^{\circ} \mathrm{C}$, saturación de oxígeno 88\% (FiO2 57\%), pulmones con estertores crepitantes, edema con fóvea en extremidades.
En complementarios se reportaron glucemia de $568 \mathrm{mg} / \mathrm{dl}$, cetonuria de +++, en gasometría acidosis metabólica compensada, urea 102.5 $\mathrm{mg} / \mathrm{dl}$, creatinina $2.38 \mathrm{mg} / \mathrm{dl}$, PCR-RT positivo para COVID-19. Se inició manejo con hidratación parenteral, oxígeno suplementario, insulina de acción rápida en infusión intravenosa, con lo que se resolvió cetoacidosis diabética. Paciente metabólicamente compensado; sin embargo, persistió la necesidad de ventilación mecánica no invasiva, paciente falleció por insuficiencia respiratoria grave debido a SARS-CoV-2.

Caso 4: mujer de 33 años, con antecedente de DM2 (tiempo de evolución desconocido) referida por insuficiencia respiratoria aguda, neumonía por SARS-CoV-2 y CAD. Al examen físico se encontró consciente y orientada, pulso de 108 latidos minuto, tensión arterial de 105/80 mmHg, temperatura de $36^{\circ} \mathrm{C}$, mucosa oral seca, mala mecánica respiratoria, 35 respiraciones por minuto y saturación de oxígeno 87\% (FiO2 0.60), cianosis, frémito aumentado en $2 / 3$ inferiores de campo pulmonar derecho, estertores crepitantes basales. En complementarios se reportó anemia moderada, glucosa: $251.5 \mathrm{mg} / \mathrm{dl}$, cetonuria de ++, osmolaridad efectiva $313 \mathrm{mOsm} / \mathrm{kg}$, gasometría arterial $\mathrm{pH} 7.24$, bicarbonato $13.7 \mathrm{mmol} / \mathrm{L}$, exceso de base $-12.6 \mathrm{mmol} / \mathrm{L}$, anión gap aumentado. PCR-RT para SARS-CoV-2 positivo. Paciente se trasladó a unidad de cuidados intensivos por descompensación metabólica y para manejo invasivo de vía aérea. Se inició protocolo con infusión de insulina, hidratación parenteral y antibioticoterapia empírica; sin embargo, paciente falleció por insuficiencia respiratoria y descompensación metabólica. 


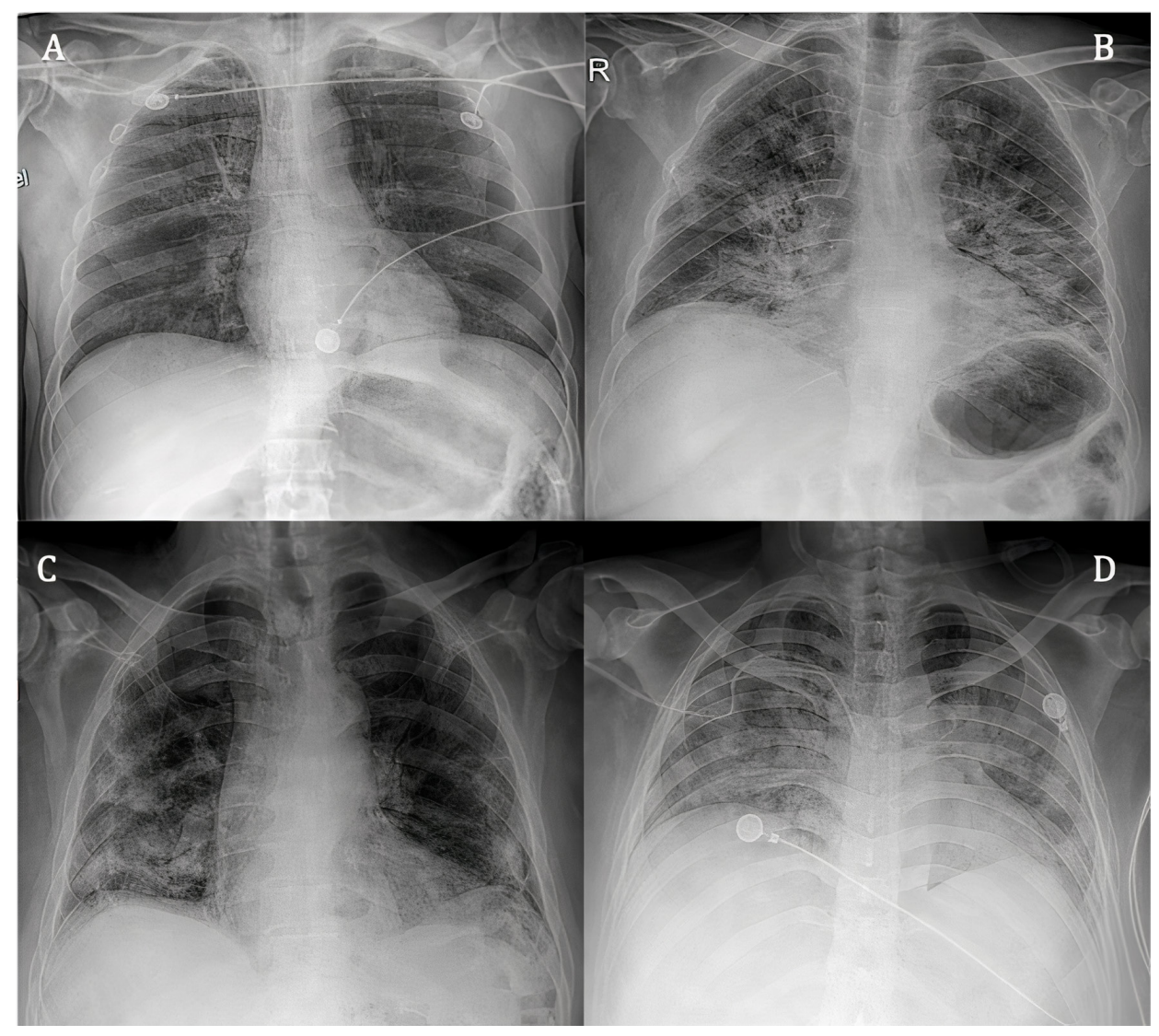

Imagen $\mathrm{N}^{\circ} 1$

Radiografías de tórax. A. Caso 1, redistribución de flujo (cefalización), opacidad pulmonar apical izquierda, perihiliar bilateral y engrosamiento peribronquial bilateral. B. Caso 2, opacidades pulmonares difusas de predominio basal y perihiliar bilateral. C. Caso 3, opacidades pulmonares en tercio medio e inferior de pulmón derecho, opacidad basal y periférica en pulmón izquierdo. D. Caso 4, opacidades pulmonares difusas y bilaterales.

\section{DISCUSIÓN}

Según la OMS en el mundo existen cerca de 422 millones de personas con DM y son consideradas un grupo vulnerable en la pandemia por COVID-198. En nuestro país según el Instituto Nacional de Estadísticas y Censos (INEC) en el año 2019, DM fue la segunda causa de muerte en adultos mayores ${ }^{9}$. La frecuencia de pacientes hospitalizados con DM y COVID-19 es alta y tiene un gran impacto en los sistemas sanitarios ${ }^{10}$, con una mortalidad de tres veces más que los pacientes no diabéticos ${ }^{11}$. Los mecanismos fisiopatológicos involucrados en la susceptibilidad a la infección por SARS-CoV-2 en DM son varios e involucran al uso de fármacos que incrementan la expresión de la enzima convertidora de angiotensina 2 (inhibidores de la enzima convertidora de angiotensina 2, estatinas, hipoglucemiantes orales), la presencia de inmunodeficiencia y el incremento de los niveles de furina (proteasa de membrana que facilita la entrada de COVID-19) ${ }^{12}$. La estrecha relación de estas dos enfermedades puede generar un círculo vicioso de deterioro, con alteración del perfil glucémico que compromete la respuesta inmune, genera un ambiente proinflamatorio y contribuye a una expresión clínica grave ${ }^{13}$.(Ver Figura $\mathrm{N}^{\circ} 1$ ).

El uso de glucocorticoides (GC) en pacientes con antecedente de DM oDM de nueva aparición puede precipitar hiperglucemia y en casos con CAD y EHNC dificultar su manejo o incluso prolongar el tiempo de su resolución. Sin embargo, son parte importante en el manejo de COVID-19 al haber demostrado disminuir la mortalidad en un tercio de pacientes con ventilación mecánica invasiva (VMI) y en una quinta parte de los pacientes que reciben oxigenoterapia sin $\mathrm{VMI}^{14}$. 
El caso 1 se presentó como debut de DM con EHNC asociada a COVID-19, la cual requirió manejo hospitalario. Para explicar el desarrollo de DM de novo en COVID-19 existen varios mecanismos como la hipótesis del mimetismo molecular; es decir, la producción de autoanticuerpos contra las células $B$ del páncreas luego de una infección viral; también están la destrucción directa de los islotes celulares del páncreas por SARS-CoV-2 y el incremento en los niveles de angiotensina II, todo lo cual genera una menor producción de insulina y el riesgo de desarrollar $\mathrm{DM}^{3,15}$, estos dos últimos mecanismos forman parte de la hipótesis de la bidireccionalidad entre DM y COVID-19 que explica la susceptibilidad de desarrollar COVID-19 en DM y de manera inversa el cómo adquirir COVID-19 puede desencadenar DM de novo ${ }^{16}$.(Ver Figura $N^{\circ} 2$ ).

\section{CONCLUSIÓN}

Es importante comprender la asociación estrecha entre DM y COVID-19 y como esta tiene un gran impacto en los sistemas sanitarios del país, es fundamental para el clínico tener una sospecha alta, ya que la presentación clínica puede ser muy variable, desde un cuadro típico a uno muy inespecífico $\mathrm{o}$ incluso presentarse como un trastorno metabólico nuevo y grave sin un antecedente previo de DM como se ha demostrado en uno de los casos, lo cual servirá para instaurar un diagnóstico correcto y tratamiento oportuno mejorando en gran medida el pronóstico de los pacientes.

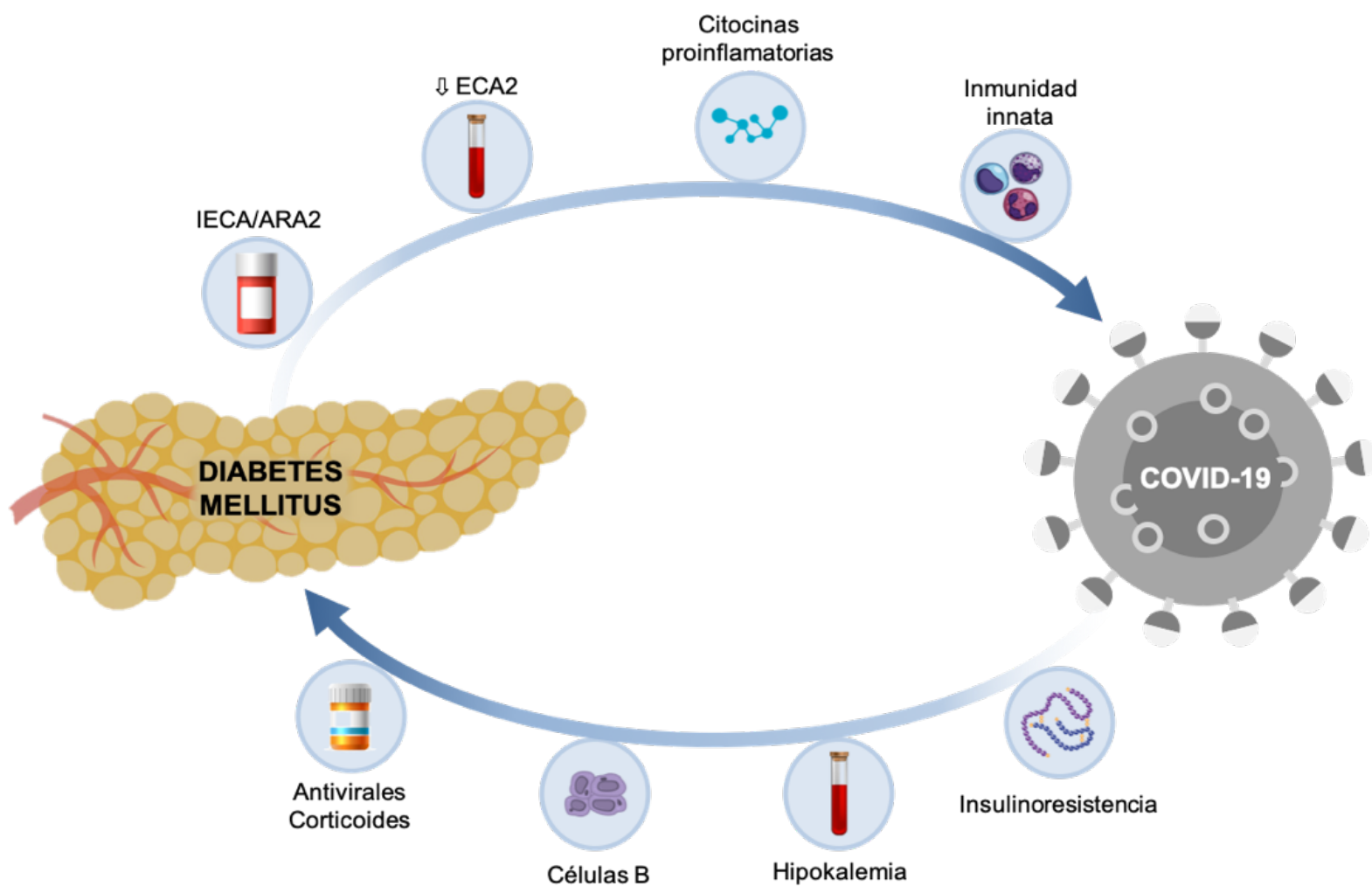

Figura $\mathrm{N}^{\circ} 1$

En DM la infección por SARS-CoV-2 puede ser grave y se debe a varios factores como: 1. El uso de fármacos del tipo inhibidores de la enzima convertidora de angiotensina 2 (IECA) y antagonistas de los receptores de angiotensina (ARA) que aumentan la expresión de la enzima convertidora de angiotensina 2 (ECA2) y que SARS-CoV-2 usa como receptor para su ingreso celular. 2. Una baja regulación de la ECA2 no permite proteger al huésped del daño pulmonar. 3. Liberación exagerada de citocinas proinflamatorias (IL-6, IL-1b, TNF-a). 4. Alteración de la respuesta inmune innata. COVID-19 a su vez empeora los niveles de glucemia en DM por los siguientes mecanismos: 1. El uso de fármacos que pueden generar hiperglucemia (antivirales, glucocorticoides). 2. Daño directo de células B. 3 y 4. Insulinoresistencia por la liberación de citocinas, fetuina A e hipokalemia ${ }^{13}$. 
A

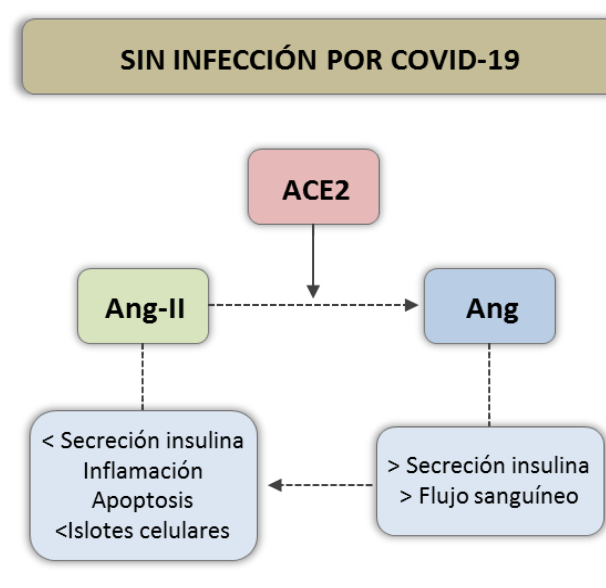

B

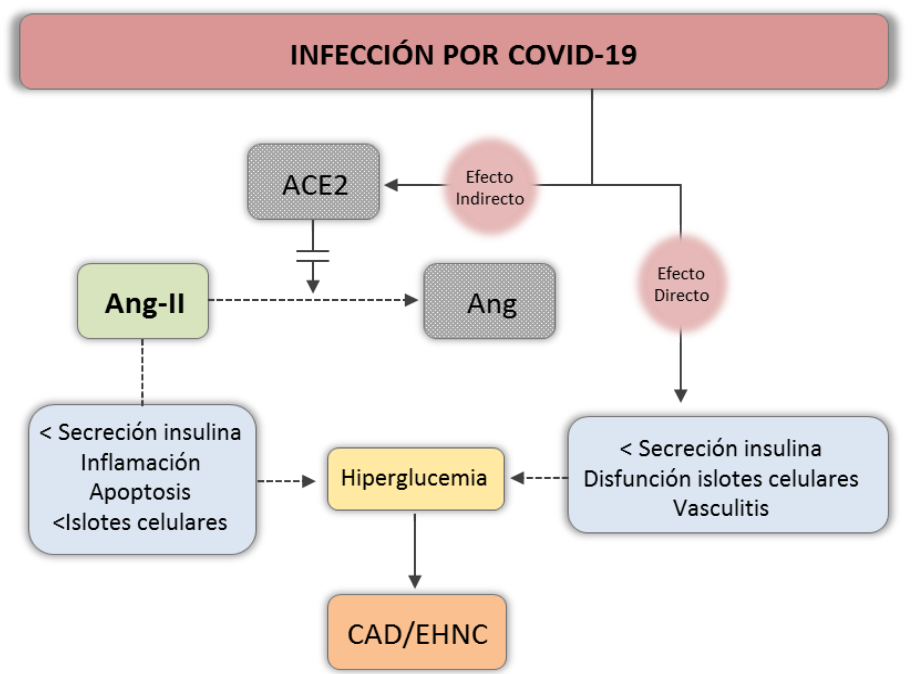

Figura $\mathrm{N}^{\circ} 2$

A. Los receptores para la ACE2 sirven de receptores funcionales para la proteína S de SARS-CoV -2, estos se expresan en varios órganos incluido el páncreas, ACE2 degrada la Ang-II en Ang, Ang II puede disminuir la secreción de insulina (lo cual se traduce en hiperglucemia), generar inflamación, apoptosis y reducir el número de islotes celulares, efecto que es contrarrestado por la Ang al incrementar la secreción de insulina y el flujo sanguíneo. B. En el caso de infección por COVID-19, su unión a los ACE2 genera una baja en la regulación de ACE2, permitiendo que predomine el efecto de Ang II (efecto indirecto); además SARS-CoV-2 puede provocar disfunción de los islotes celulares, disminución en la producción de insulina e inflamación vascular (efecto directo), todo lo cual lleva a una crisis aguda de hiperglucemia con el riesgo de desarrollar complicaciones agudas tipo CAD y EHNC. 3,15 COVID-19: enfermedad por SARS-CoV-2. ACE2: enzima convertidora de angiotensina 2. Ang-II: angiotensina 2. Ang: angiotensina. CAD: cetoacidosis diabética. EHNC: estado hiperosmolar no cetósico.

\section{ASPECTOS BIOÉTICOS}

El presente estudio se efectuó con la aprobación del Comité de Bioética Institucional en donde se realizó la investigación, con total confidencialidad de los datos personales de los pacientes y con los respectivos consentimientos informados.

\section{INFORMACIÓN DE LOS AUTORES}

- Sacoto Flores Goethe Salomón. Médico. Especialista en Medicina Interna. Hospital Homero Castanier Crespo. Clínica. Hospitalización COVID-19. Cuenca-Azuay-Ecuador.

e-mail: goethe.sacoto@ucuenca.edu.ec

ORCID: https://orcid.org/ 0000-0002-8114-0114

- Sánchez Coraizaca Galo Dario. Médico. Hospital Homero Castanier Crespo. Clínica. UCI COVID-19. Azogues-Cañar-Ecuador e-mail: gsanchezc2112@gmail.com ORCID: https://orcid.org/0000-0003-4695-3096
- Cajilema Acero Luis Andrés. Médico. Hospital Homero Castanier Crespo. Clínica. Hospitalización COVID-19. El Tambo-CañarEcuador.

e-mail: andrescajilema@gmail.com

ORCID: https://orcid.org/0000-0002-0085-5426

- Sanango Jara María Ximena. Médica. Hospital Homero Castanier Crespo. Clínica. Hospitalización COVID-19. Azogues-Cañar-Ecuador.

e-mail: ximena.sanangojara@gmail.com

ORCID: https://orcid.org/0000-0003-2207-3131

\section{CONTRIBUCIÓN DE LOS AUTORES}

Todos los autores contribuyeron en la concepción y diseño del trabajo con el correspondiente análisis e interpretación de los datos, redacción y revisión crítica del manuscrito, aprobación de la versión final y están en capacidad de responder de todos los aspectos del artículo. 


\section{CONFLICTO DE INTERESES}

Los autores declaran no tener conflicto de intereses.

\section{FUENTES DE FINANCIAMIENTO}

Autofinanciado

\section{AGRADECIMIENTO}

Agradecemos a todo el personal de nuestra institución por su inexorable esfuerzo en la lucha contra la pandemia y a todos los pacientes con COVID-19 en especial a los que fueron objeto de nuestro estudio.

\section{BIBLIOGRAFÍA}

1. Zhu N, Zhang D, Wang W, Li X, Yang B, Song $\mathrm{J}$, et al. A novel coronavirus from patients with pneumonia in China, 2019. N. Engl. J. Med. 382, 727-733. doi: 10.1056/ NEJMoa2001017.

2. Guan WJ, Liang WH, Zhao Y, Liang HR, Chen ZS, Li YM, et al. Comorbidity and its impact on 1590 patients with COVID-19 in China: a nationwide analysis. Eur Respir J. 2020;55(5): 2000547. doi: 10.1183/13993003.005472020.

3. Viswanathan $V$, Puvvula $A$, Jamthikar $A D$, Saba L, Johri AM, Kotsis V, et al. World J Diabetes. 2021 Mar 15;12(3):215-237. doi: 10.4239/wjd.v12.i3.215.

4. Guo W, Li M, Dong Y, Zhou H, Zhang Z, Tian C. Diabetes is a risk factor for the progression and prognosis of COVID-19. Diabetes Metab Res Rev. 2020;36(7):e3319. doi: 10.1002/ dmrr.3319.

5. Li J, Wang X, Chen J, Zuo X, Zhang H, Deng A. COVID-19 infection may cause ketosis and ketoacidosis. Diabetes Obes Metab. 2020 Oct;22(10):1935-1941. doi: 10.1111/ dom.14057.

6. Zhou F, Yu T, Du R, Fan G, Liu Y, Liu Z. Clinical course and risk factors for mortality of adult inpatients with COVID-19 in Wuhan, China: a retrospective cohort study. Lancet. 2020 Mar 28;395(10229):1054-1062. doi: 10.1016/S0140-6736(20)30566-3.
7. Kitabchi AE, Umpierrez GE, Miles JM, Fisher JN. Hyperglycemic crises in adult patients with diabetes. Diabetes Care. 2009 Jul;32(7):1335-43. doi: 10.2337/dc09-9032.

8. Diabetes [Internet]. WHO.Int. [citado 1 abril 2021]. Disponible en: https://www.who.int/ news-room/fact-sheets/detail/diabetes

9. Estadísticas de defunciones generales en el Ecuador [Internet]. Gob.ec. [citado 1 abril 2021]. Disponible en: https://www. ecuadorencifras.gob.ec

10. Oliveira E, Parikh A, Lopez-Ruiz A, Carrilo M, Goldberg J, Cearras M. ICU outcomes and survival in patients with severe COVID-19 in the largest health care system in central Florida. PLoS One. 2021 Mar 25;16(3):e0249038. doi: 10.1371/journal. pone. 0249038

11. Roncon L, Zuin M, Rigatelli G, Zuliani G. Diabetic patients with COVID-19 infection are at higher risk of ICU admission and poor shortterm outcome. J Clin Virol 2020;127:104354. https://doi.org/10.1016/j.jcv.2020.104354.

12. Fernandez $C$, Rysä J, Almgren $P$, Nilsson $\mathrm{J}$, Engström G, Orho-Melander M. Plasma levels of the proprotein convertase furin and incidence of diabetes and mortality. J Intern Med. 2018; 284: 377-387. doi: 10.1111/ joim. 12783

13. Pal R, Bhadada SK. COVID-19 and diabetes mellitus: An unholy interaction of two pandemics. Diabetes Metab Syndr. 2020 Jul-Aug;14(4):513-517. doi: 10.1016/j. dsx.2020.04.049.

14. Rayman, G., Lumb, A. N., Kennon, B., Cottrell, C., Nagi, D., Page, E., Thomas, S. (2020). Dexamethasone therapy in COVID $\square 19$ patients: implications and guidance for the management of blood glucose in people with and without diabetes. Diabetic Medicine. doi:10.1111/dme.14378

15. Fignani D, Licata G, Brusco N, Nigi L, Grieco GE, Marselli L. SARS-CoV-2 Receptor Angiotensin I-Converting Enzyme Type 2 (ACE2) Is Expressed in Human Pancreatic $\beta$-Cells and in the Human Pancreas Microvasculature. Front Endocrinol (Lausanne). 2020 Nov 13;11:596898. doi: 10.3389/fendo.2020.596898. 
16. Singh AK, Gupta R, Ghosh A, Misra A. Diabetes in COVID-19: Prevalence, pathophysiology, prognosis and practical considerations. Diabetes Metab Syndr 2020; 14: 303-310. doi: 10.1016/j.dsx.2020.04.004 